\title{
Occult prostatic adenocarcinoma presenting as a ureteric tumor and expressing KIT and PDGFRA
}

\author{
Tadashi Terada* \\ Department of Pathology, Shizuoka City Shimizu Hospital, Shizuoka, Japan
}

Received: February 28, 2016

Accepted: April 4, 2016

Online Published: April 12, 2016

DOI: $10.5430 /$ crcp.v3n4p11

URL: http://dx.doi.org/10.5430/crcp.v3n4p11

\begin{abstract}
Prostatic carcinoma (PC) may present multiple metastases of unknown primary (most commonly bone metastases), the occult PC. PC expressing KIT and PDGRA has not been reported. There have been no reports of occult PC manifesting as a ureter tumor. An 83-year-old man presented with flank pain. Imaging showed a ureter tumor. Ureteronephrectomy was performed. The author diagnosed the ureteric tumor as high-grade urothelial carcinoma, Postoperative imaging showed multiple tumors in liver and lymph nodes. The clinical diagnosis was hepatocellular carcinoma and cirrhosis, and the patient received radiofrequency ablation (RFA). The patient further presented hematuria, and cystscopy revealed an elevated tumor of urinary bladder, and TUR-BT was performed. Histologically, TUR-BT specimens show monotonous medullar proliferation of high-grade malignancy arranged in solid nests and acinar formations. Immunohistochemically, tumor cells were positive for PSA, $\alpha$-methyl CoA racemase (AMACR), cytokeratin (CK) AE1/3, CK CAM5.2, CK8, CK18, CK19, CEA, synaptophysin, KIT, PDGFRA, p53 and Ki-67 (labeling index $=90 \%$ ). No mutations of KIT and PDGFRA were noted. Since PSA and AMACR were positive, the definite diagnosis of PC (Gleason 9) metastatic to multiple sites including ureter and bladder was made.
\end{abstract}

Key Words: Prostate, Occult carcinoma, Adenocarcinoma, KIT, PDGFRA

\section{INTRODUCTION}

Prostatic carcinoma (PC) is one of the most common malignancies in male in the world. ${ }^{[1]}$ In western countries, $\mathrm{PC}$ is the most common malignancy in men. In Japan, the most common malignant tumor is lung carcinoma. However, the prevalence of $\mathrm{PC}$ is strikingly increasing probably due to the western style of food intake. PC is occasionally occult; the metastases of PC manifest as initial lesions. In most of such occult PC, most common metastatic sites are bones, but all organs can be the sites of main initial lesion of occult PC..$^{[1]}$

Primary ureter tumors are very rare. Most of the primary ureter tumor is urothelial carcinoma, ${ }^{[2]}$ though some case of small cell carcinoma and squamous cell carcinoma are reported ${ }^{[3,4]}$ Secondary tumor of ureter is extremely rare. ${ }^{[2]}$ The author's search of the world literature revealed only six cases of PC metastatic to ureter. ${ }^{[5-10]}$ In addition, there have been no reports of occult PC initially manifesting as ureter tumor.

There have been no reports of prostatic adenocarcinoma (PA) with strong protein expressions of KIT and PDGFRA. Also, there have been no studies of mutational analysis of KIT and PDGFRA genes in PA with strong expressions of KIT and PDGFRA proteins. In PC, almost all PC is acinar adenocarcoinoma and ductal carcinoma is very rare. ${ }^{[2]}$ Herein reported is a very rare case of occult PA of Gleason score 9 with strong expression of KIT and PDGFRA and initially

\footnotetext{
*Correspondence: Tadashi Terada; Email: piyo0111jp@yahoo.co.jp; Address: Department of Pathology, Shizuoka City Shimizu Hospital, Miyakami 1231 Shimizu-Ku, Shizuoka 424-8636, Japan.
} 
manifesting as a ureter tumor. A molecular analysis of KIT and PDGFRA genes was made.

\section{CASE REPORT}

An 83-year-old man presented with flank pain. Imagings showed right hydronephrosis and a ureter tumor. Blood laboratory data showed no significant changes except positive antibody to hepatitis $\mathrm{C}$ virus (HCV). The tumor markers were investigated in only AFP and PIVKA-II, both of which were within normal ranges. The urologists suspected a ureter tumor. Clinical cytology of right ureter urine showed atypical cells suspicious of urothelial carcinoma (UC). Operation was planned; preoperative imaging examinations by radiologists could not identify tumors of other locations but a retrospective survey (done 3 months after) of the imaging showed suspicious small tumors in the liver and para-aortic lymph nodes.

Therefore, right uretero-nephrectomy was performed. There was a right ureter tumor at the distal part and hydronephrosis (see Figure 1). The tumor of right ureter measured $3 \mathrm{~cm} \times 3 \mathrm{~cm} \times 2 \mathrm{~cm}$. Microscopically, the mucosa, submucosa, muscle layer and advantitia were occupied by a medullary undifferentiated carcinoma cells (see Figure 2A). No apparent differentiations were seen. Because the ureteric tumor did not show significantly specific features and the most common histological type of ureteric tumors is urothelial carcinoma (UC), the author diagnosed the ureter tumor as high-grade non-papillary urothelial carcinoma. No Immunohistochemical study was done at that time; but later retrospective immunohistochemical study showed the same findings as those of the bladder tumor.

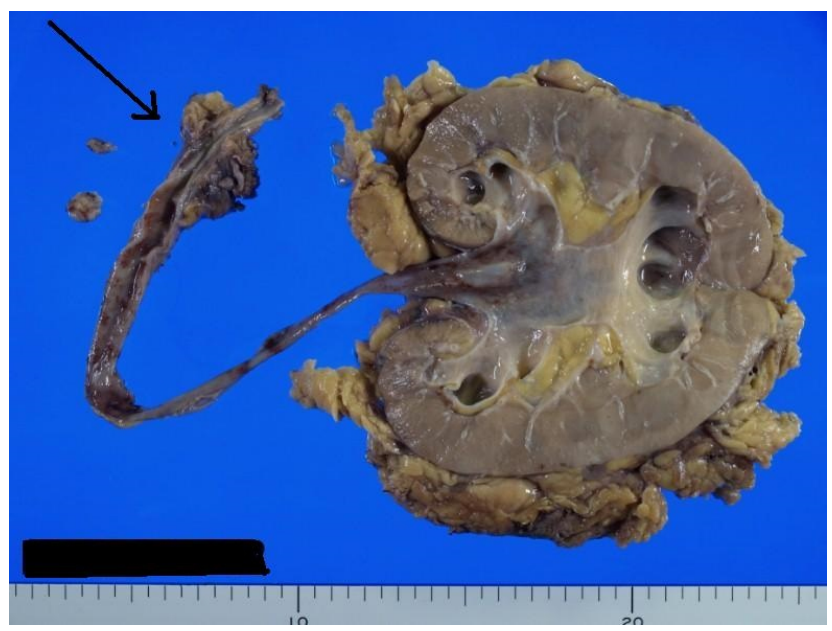

Figure 1. The resected right kidney and ureter. The ureter has $3 \mathrm{~cm} \times 3 \mathrm{~cm} \times 2 \mathrm{~cm}$ tumor (arrow). The kidney shows hydronephrosis.
Postoperative imaging showed multiple tumors of the liver and abdominal lympho-adenopathy. The radiologists' diagnosis was hepatocellular carcinoma (HCC) and cirrhosis related to $\mathrm{HCV}$, and the patient received radiofrequency ablation (RFA) therapy for HCC. No biopsies of the liver and abdominal lymph nodes were made; the clinicians and radiologists firmly believed that the tumor was HCC and its lymph node metastasis. Clinicians and radiologists seemed not to discuss the possible links among the ureteric tumor, liver tumors, and lymphadenopathy.

Three months layer, the patient complained of hematuria, and cystscopy was performed. It revealed an elevated tumor of the urinary bladder (UB), and transurethral resection of bladder tumor (TUR-BT) was performed. Histologically, the TUR-BT specimens show monotonous medullar proliferation of high-grade malignant cells arranged in solid nests, and showed very focally acinar formations, which were not seen in the ureter tumor. The nuclei of the tumor cells were vesicular and hyperchromatic, and contained prominent nucleoli. The appearances were those of poorly differentiated adenocarcinoma.

Immunohistochemically, the tumor cells were positive for PSA (see Figure 2B), $\alpha$-methyl CoA racemase (AMACR) (see Figure 2C), cytokentin (CK) AE1/3, CK CAM5.2, CK8, CK18, CK19, CEA, synaptophysin, KIT (see Figure 2D), PDGFRA (see Figure 2E), p53, and Ki-67 (labeling index = 90\%) (see Figure 2F). They were negative for CK34BE12, CK5, CK6, CK7, CK20, NSE, vimentin, p63, S100 protein, $\alpha$-smooth muscle actin, CD34, CA19-9, chromogranin, and NCAM.

Since PSA and AMACR were positive, the definite diagnosis of prostatic adenocarcinoma (PA) metastatic to UB was made. Other histological and Immunohistochemical features are compatible with primary occult PC. Serum PSA level was firstly measured after the correct pathological diagnosis of UB, and the serum PSA was very high (112 ng/ml). Later prostatic biopsy revealed poorly differentiated PA of Gleason 9 score positive for PSA and AMACR. The retrospective immunohistochemical investigation of the ureter tumor showed positive reactions for PSA and AMACR, and therefore it was found that the ureter tumor was a metastasis of occult PA. Other immunohistochemical data of the ureter tumor were the same as those of the UB tumor. Although not examined pathologically, the liver tumors and lymphadenopathy seemed metastases from the occult PC. A molecular genetic analysis of KIT gene (exons 9, 11, 13, and 17) and PDGFRA gene (exons 12 and 18) was performed, and it identified no mutations. The treatment included LH-RH agonists, preventive bisphosphonate, and cisplatin-based chemotherapy. 

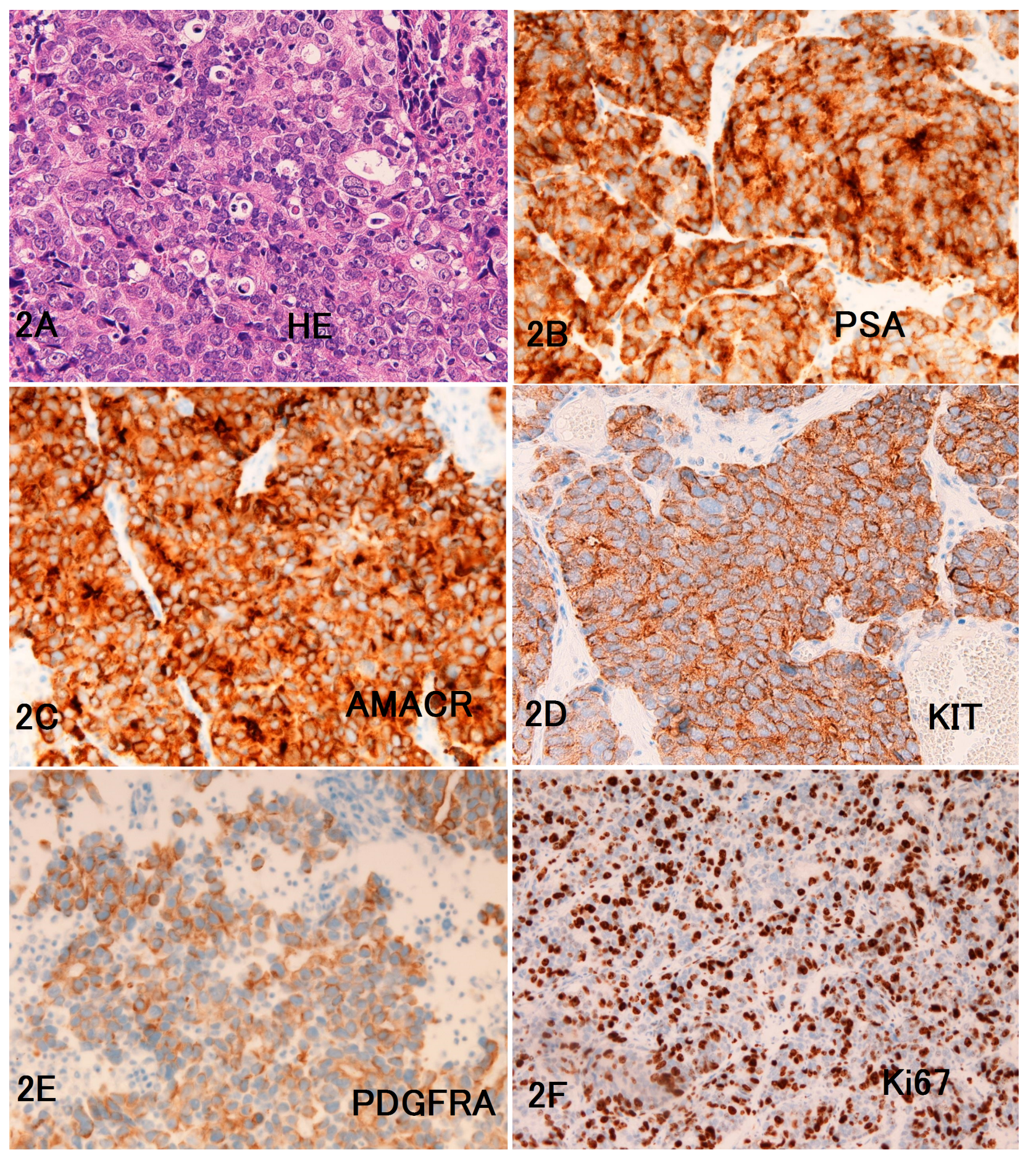

Figure 2. A: Histological features of the ureter tumor showing poorly differentiated or undifferentiated carcinoma. (Later is was found to be Gleason 9 prostatic adenocarcinoma). HE, $\times 200$. B-F: Immunohistochemical features. The tumor cells are positive for PSA and AMACR, both with a membranous and cytoplasmic pattern. The tumor cells were positive for KIT (CD117) (D) and PDGFRA (E), both with a membranous pattern. The tumor cells show high Ki67 labeling index with a nuclear pattern. A-F: $\times 200$.

\section{Discussion}

The current case is interesting in two aspects. Others are less interesting although many new findings are seen. The first point is that the present PC expressed strongly KIT and Published by Sciedu Press
PDGFRA proteins. No mutations of KIT and PDGFRA were seen in the present case. The author examined numerous cases of tumor with KIT and PDGFRA protein. Of these, tumors of mutations in KIT or PDGFRA were seen only in 
GIST, e-GIST, melanoma, and germ cell tumors. Other tumors positive for KIT or PDGFRA, which includes small cell lung carcinoma, extra-pulmonary SCC, basal cell carcinoma, adenoid cystic carcinoma, myoepithelioma, mastocytoma, and other tumors, were consistently negative for KIT and PDGFRA gene mutations. The present study firstly reported strong expressions of KIT and PDGFRA in ordinary PC with high-grade atypia of Gleason 9. No gene mutations of KIT and PDGFRA were found in this case.

The second important point is that the present occult PC firstly presented a ureter tumor. The clinical and diagnostic cytology suggested the primary ureter UC. The pathological diagnosis of the ureteric tumor was mistake; he diagnosed the ureter tumor as high-grade (grade 3) undifferentiated UC. The author regrets that if at that time an immunohistochemical study was performed, the diagnosis of PC metastatic to the ureter can be made. It suggests that although immunohistochemical and molecular studies take much time, they should be done when pathologists encountered unusual tumors.

Primary tumor of the ureter is very rare. ${ }^{[2]}$ Almost all of them are UC. In addition, secondly or metastatic tumor of the ureter is very rare. Further, $\mathrm{PC}$ metastatic to the ureter is exceptional; only six cases have been reported in the world literature. ${ }^{[5-10]}$ Moreover, there have been no cases of PC firstly manifested as a ureter tumor. Pathologists must keep in mind that such rare conditions may occur in any cancers.

The present patient is an old man and is in the advanced stage (Stage 4) of PC. Imatinib mesylate is a molecular targeting drugs binding to KIT. Imatinib is now regarded as effective in GIST, e-GIST and some hematopoitic malignancies. If activating or gain-of-function mutations were found, imatinib mesylate may be effective. Although not tried, imatinib may be non-effective in the present case because of no mutations in KIT and PDGFRA genes. Of course Imatinib (Gleevec) is not used for treatment of PC. Here, the author only examined the KIT and PDGFRA status of a case of PC. In this patient in Stage 4, the patient was treated with LH-RH agonists and cisplatin-based chemotherapy, a variant of NCI regimen for Stage 4 prostatic cancers. Bisphosphonate is also given for prevention of bone metastases.

\section{CONFlicts OF InTEREST Disclosure}

The author declares no conflict of interest.

\section{REFERENCES}

[1] Epstein JL, Algaba F, Allsbrook Jr WC, et al. Tumours of the prostate. Acinar adenocarcinoma, In: Moch H, Humphrey PA, Ulbright TM, Reuter VE eds: World Health Organization Classification of Tumours. Pathology and genetics of tumours of the urinary system and male genital organs. Lyon: IARC Press; 2016. 135p. PMid: 26897585.

[2] Lopez-Beltran A, Sauter G, Gasser T, et al. Tumours of the renal pelvis and ureter. In: Moch H, Humphrey PA, Ulbright TM, Reuter VE eds: World Health Organization Classification of Tumours. Pathology and genetics of tumours of the urinary system and male genital organs. Lyon: IARC Press; 2016. 93p.

[3] Terada T. Primary small cell carcinoma of the ureter: a case report involving immunohistochemical and molecular genetic analyses of KIT and PDGFRA genes. Pathology. 2010; 42: 101-2. PMid: 20025496. http://dx.doi.org/10.3109/00313020903443018

[4] Terada T. Synchronous squamous cell carcinoma of the kidney, squamous cell carcinoma of the ureter, and sarcomatoid carcinoma of the urinary bladder: a case report. Pathol Res Pract. 2010; 206: 379-83. PMid: 19766408. http://dx.doi.org/10.1016/j.prp. 2009. 07.021
[5] Jallad S, Turo R, Kimuli M, et al. Ureteric stricture: an unusual presentation of metastatic prostate adenocarcinoma. Ann R Coll Surg Engl. 2012; 94: e213-4. PMid: 23031752. http://dx.doi.org/1 $0.1308 / 003588412 \times 13373405385971$

[6] Schneider S, Popp D, Denzinger S, et al. A rare location of metastasis from prostate cancer: hydronephrosis associated with ureteral metastasis. Adv Urol. 2012; 2012: 656023. PMid: 21912541. http://dx.doi.org/10.1155/2012/656023

[7] Maeda N, Yoshida T. Metastatic tumor of renal pelvis and ureter from prostatic cancer: a case report. Hinyokika Kiyo. 1999; 45: 273-5.

[8] Yonneau L, Lebret T, Hervé JM, et al. Isolated ureteral metastasis of prostatic adenocarcinoma. Apropos of a case. Prog Urol. 1999; 9: 118-21.

[9] Hulse CA, O'Neill TK. Adenocarcinoma of the prostate metastatic to the ureter with an associated ureteral stone. J Urol. 1989; 142: 1312-4. PMid: 2810518.

[10] Benejam R, Carroll TJ, Loening S. Prostate carcinoma metastatic to ureter. Urology. 1987; 29: 325-7. http://dx.doi.org/10.1016 /0090-4295 (87) 90084-7 